\title{
DISERTACIONES
}

ENSAYOS

Anuario electrónico de estudios en Comunicación Social

ISSN: $1856-9536$

Doi: dx.doi.org/10.12804/disertaciones.09.02.2016.01

Volumen 9, Número 2 / julio-diciembre 2016

Versión PDF para imprimir desde

http://revistas.urosario.edu.co/index.php/disertaciones

Para citar este artículo: Fuentes, R. (2016). Cuatro décadas de internacionalización académica en el campo de estudios de la comunicación en América Latina. Anuario Electrónico de Estudios en Comunicación Social “Disertaciones", 9(2), 8-26. Doi: dx.doi.org/10.12804/disertaciones.09.02.2016.01

\section{CUATRO DÉCADAS DE INTERNACIONALIZACIÓN ACADÉMICA EN EL CAMPO DE ESTUDIOS DE LA COMUNICACIÓN EN AMÉRICA LATINA}

Four Decades of Internationalization in Latin American Communication Studies

Quatro décadas de internacionalização acadêmica no campo de estudos da comunicação na América Latina

Fuentes Navarro, Raúl. ITESO (México)

raul@iteso.mx

Fecha de recibido: 22 de febrero de 2016

Fecha de aceptado: 13 de abril de 2016

\section{RESUMEN}

Este ensayo tiene el objetivo de proponer una relectura de la historia de la institucionalización académica del estudio de la comunicación en América Latina que, dentro de un marco conceptual y contextual en plena construcción colectiva internacional, aporte información puntual y una interpretación renovada sobre el papel que en esta historia han desempeñado la Asociación Latinoamericana de Investigadores de la Comunicación (ALAIC) y la Federación Latinoamericana de (Asociaciones de) Facultades de Comunicación Social (FELAFAcS) durante las cuatro décadas más recientes, en términos de la internacionalización del campo. El desarrollo de esta relectura utiliza la recuperación de interpretaciones encontradas, sostenidas y publicadas desde los años ochenta hasta la actualidad dentro y fuera de la región, pues no puede seguirse considerando linealmente la vigencia de una sola forma de Pensamiento Crítico Latinoamericano en Comunicación. 


\title{
DISERTACIONES
}

ENSAYOS

Anuario electrónico de estudios en Comunicación Social

ISSN: 1856-9536

Doi: dx.doi.org/10.12804/disertaciones.09.02.2016.01

Volumen 9, Número 2 / julio-diciembre 2016

Versión PDF para imprimir desde

http://revistas.urosario.edu.co/index.php/disertaciones

Palabras clave: investigación de la comunicación, campo académico, América Latina, institucionalización, internacionalización.

\begin{abstract}
The main purpose of this paper is to propose a reinterpretation of the history of academic institutionalization of communication studies in Latin America, within a conceptual and contextual framework presently under international collective construction, seeking to supply precise information and a renewed understanding of the role the Latin American Association of Communication Researchers (ALAIC) and the Latin American Federation of (Associations of) Faculties of Social Communication (FELAFACS) have played for the four most recent decades, to foster the internationalization of the field. The development of this proposed reinterpretation applies the recovery of conflicting versions, supported and published from the eighties until today, inside and outside the region, because a linear consideration of the validity of a single form of Latin American Communication Critical Thought is no longer acceptable.
\end{abstract}

Keywords: Communication Research, Academic Field, Latin America, Institutionalization, Internationalization.

\section{RESUMO}

Este ensaio tem o objetivo de propor uma releitura da história da institucionalização acadêmica do estudo da comunicação na América Latina que, dentro de um marco conceitual e contextual em completa construção coletiva internacional, aporte informação pontual e uma interpretação renovada sobre o papel que nesta história têm desempenhado a Associação Latino-americana de Investigadores da Comunicação (ALAIC) e a Federação Latino-americana de (Associações de) Faculdades de Comunicação Social (FELAFAcs) durante as quatro décadas mais recentes, em termos da internacionalização do campo. O desenvolvimento desta releitura utiliza a recuperação de interpretações encontradas, sustentadas e publicadas desde os anos oitenta até a atualidade dentro e fora da região, pois não pode seguir-se considerando linearmente a vigência de uma só forma de Pensamento Crítico Latino-americano em Comunicação.

Palavras-chave: Investigação da comunicação, campo acadêmico, América Latina, institucionalização, internacionalização. 


\section{DISERTACIONES}

Ensayos

Anuario electrónico de estudios en Comunicación Social

ISSN: $1856-9536$

Doi: dx.doi.org/10.12804/disertaciones.09.02.2016.01

Volumen 9, Número 2 / julio-diciembre 2016

Versión PDF para imprimir desde

http://revistas.urosario.edu.co/index.php/disertaciones

Una de las más llamativas características de las investigaciones en materia de comunicación en América Latina - un poco en contraste con lo que ocurre en Europa y en otras partes del mundo- es la notable intercomunicación que existe entre los investigadores, los proyectos de investigación cooperativa y la conexión entre diversas organizaciones, institutos, publicaciones y facultades (White, 1989, p. 44).

\section{Introducción}

Leída más de veinticinco años después, esta apreciación de Robert White, publicada en el primer número especial (el 19) que dedicó la revista española Telos (dirigida por Enrique Bustamante) a revisar el estado de la cuestión en cuanto a "Comunicación, cultura y nuevas tecnologías; teoría, políticas e investigación" en América Latina, llama la atención por el énfasis en los lazos de solidaridad e interconexiones continentales entre instituciones e individuos, lo que White (1989) Ilamó "amigocracia", una descripción afectuosa que ha sido valorada muchas veces negativamente o considerada insuficiente e ineficiente por actores latinoamericanos de este campo, incluyendo a varios de los "amigos" aludidos".

Sin pretender provocar ahora una discusión anacrónica e insustancial sobre las implicaciones de esa figura de lenguaje en la interpretación histórica de la teoría de la comunicación en América Latina, este trabajo parte de aquella observación de White para subrayar el carácter constitutivo de la intercomunicación organizada y sostenida entre actores individuales e institucionales en una región tan grande como América Latina (más de 580 millones de habitantes en 20 millones de kilómetros cuadrados), y reinterpretar la importancia que puede atribuirse a los procesos de institucionalización de los nexos académicos interpersonales, interinstitucionales e internacionales sostenidos desde hace cuatro décadas, en especial a los canalizados o conducidos por la Asociación Latinoamericana de Investigadores de la Comunicación (ALAIC, constituida en 1978 y reconstituida en 1989) y por la Federación Latinoamericana de (Asociaciones de) Facultades de Comunicación Social (FELAFACS, instituida en 1981)2.

Evidentemente, el estudio de la comunicación en América Latina no comenzó con la fundación de estas dos organizaciones ${ }^{3}$, pero puede sostenerse que, así como la configuración presente del campo académico no podría

1 En ese número de Telos (publicado en 1989), más que en otras compilaciones sobre el "Pensamiento Crítico Latinoamericano en Comunicación", es muy interesante el contraste y complementación de perspectivas cercanas y comprometidas pero finalmente "externas" como la de White o la de Philip Schlesinger (1989), con las de los latinoamericanos convocados, pues predomina en estos últimos una postura también profundamente comprometida pero fuertemente autocrítica, sobre todo en los "balances" ("impensables e irrealizables años atrás" - según Rafael Roncagliolo, coordinador del número desde Lima-) sobre la producción bibliográfica reciente (Martín-Barbero, 1989); las revistas y su contribución a la "creación de la teoría militante" (Gonzaga Mota, 1989); los centros de investigación de la comunicación (Marques de Melo, 1989); y las escuelas universitarias (Fuentes, 1989) en América Latina, es decir, sobre algunas de las concreciones principales de la institucionalización académica del campo. Este contraste de perspectivas se retomará más adelante en este texto como eventual clave interpretativa de estos procesos.

2 http://alaic.org/site/, http://felafacs.org/.

3 Desde su fundación en 1959, siguiendo una iniciativa de la Unesco, el Centro Internacional de Estudios Superiores de Periodismo (luego, Comunicación) para América Latina (CIESPAL) había ejercido una gran 


\section{DISERTACIONES}

Ensayos

Anuario electrónico de estudios en Comunicación Social

ISSN: $1856-9536$

Doi: dx.doi.org/10.12804/disertaciones.09.02.2016.01

Volumen 9, Número 2 / julio-diciembre 2016

Versión PDF para imprimir desde

http://revistas.urosario.edu.co/index.php/disertaciones

entenderse sin su intervención, tampoco pueden ubicarse adecuadamente sus inicios sin el reconocimiento bien documentado y asumido de los contextos históricos, de distintas escalas y dimensiones, en que fueron establecidas (Fuentes, 1992, 2016; Gómez-Palacio, 1989; Marques de Melo, 1988, 2015). Este texto propone así bases para una relectura del "Pensamiento Crítico Latinoamericano en Comunicación" anclada en los procesos de institucionalización de estas dos organizaciones académicas y sus múltiples contribuciones a la internacionalización del campo. El desarrollo de esta relectura utiliza la recuperación de interpretaciones encontradas al respecto, sostenidas y publicadas desde hace casi tres décadas en y sobre América Latina. Desde la misma lógica de contraste discursivo, puede recuperarse, a manera de ejemplo, la postura de Joli Jensen en el contexto del debate estadounidense en los años noventa:

Las revistas académicas y las organizaciones profesionales son los indicadores más obvios de lo que constituye el contenido y las fronteras del campo. En las reuniones y los artículos es donde se evidencian con mayor fuerza las preocupaciones sobre la unidad o la fragmentación de un campo, sus paradigmas dominantes, su estatus disciplinario y sus batallas metodológicas. ¿Por qué importa todo esto? ¿A quién le importa si aquello en lo que estamos es un campo o una disciplina o un área de investigación, o si podemos estar de acuerdo en ciertas teorías o procedimientos? Pues a nosotros nos importa porque las respuestas a estas preguntas moldean nuestro medio de vida: no solo lo que hacemos sino cómo somos percibidos por otros (Jensen, 1994, p. 76).

A pesar, entonces, de que puede caracterizarse como una "internacionalización desintegrada" (Fuentes, 2014b, p. 15), siguiendo la hipótesis de que un proceso de fragmentación o de divergencia múltiple ha sustituido, tanto en el plano epistemológico o metodológico como en el plano de la acción transformadora de los sistemas y las prácticas sociales de comunicación, a las polarizaciones típicas de otras épocas, lamentablemente sin reducir los riesgos del dogmatismo que Beltrán (1974) había identificado desde los años setenta (Fuentes, 2015), la identidad latinoamericana en los estudios de la comunicación es una referencia histórica ineludible, que donde mejor puede apreciarse es precisamente en las publicaciones, proyectos y encuentros académicos institucionalizados por ALAIC y feLAFACS a lo largo de las cuatro últimas décadas, procesos que ciertamente nos importan y nos incumben.

influencia en la conformación de un campo definido y reconocido como latinoamericano, basado en una red de académicos de diversos países capacitados en su sede de Quito, primero en cuanto a la formación profesional y luego con respecto a la investigación y otras líneas de trabajo en comunicación. No obstante su carácter de organismo internacional, CIESPAL no puede considerarse una organización académica propiamente dicha, como ALAIC y FELAFACS, promovidas y gestionadas por individuos e instituciones académicas y no por instancias gubernamentales o agencias internacionales. Además de la intensa intervención de CIESPAL, otras organizaciones contribuyeron también, desde la década de los años veinte, a establecer vínculos internacionales, relacionados con el estudio de la comunicación, tanto internos como externos a la región latinoamericana, que no son considerados en este trabajo. 


\section{DISERTACIONES}

ENSAYOS

Anuario electrónico de estudios en Comunicación Socia

ISSN: $1856-9536$

Doi: dx.doi.org/10.12804/disertaciones.09.02.2016.01

Volumen 9, Número 2 / julio-diciembre 2016

Versión PDF para imprimir desde

\section{Un marco para reconstruir la historia de la estructuración del campo académico}

Desde una perspectiva sociocultural desarrollada a lo largo de múltiples experiencias de investigación (Fuentes, 1992; 1998; 2006; 2016), se considera a la institucionalización en programas universitarios y asociaciones profesionales como la manifestación más "objetiva" de la constitución de un campo académico ${ }^{4}$, en la medida en que de esa forma las instancias del poder social asignan o reconocen un lugar específico a la producción y reproducción del conocimiento y a la formación profesional en un área determinada, e implícita o explícitamente definen la orientación y el sentido (función social) que el trabajo sobre dicha área en dicho lugar deberá de cumplir para obtener y reforzar su legitimidad. Este proceso es entonces inseparable de la profesionalización de los sujetos que, dentro de los programas establecidos, han de ejercer las prácticas académicas y articula, de maneras más o menos fuertes, la producción académica con la toma de decisiones en el área, lo cual a su vez contribuye a la legitimación del conocimiento, de las instituciones donde se cultiva y de los sujetos que lo generan.

Por ello, la extensión y la distribución de los programas en el sistema de educación superior de uno u otro país indica, al mismo tiempo, las posiciones que va adquiriendo la disciplina en el sistema en relación con otras, y las que distinguen entre sí a las instituciones universitarias en la constitución del campo, así como las redes que las articulan de ciertas maneras y no de otras. Pero, además de estos procesos de institucionalización social en establecimientos universitarios y redes de interconexión entre ellos (Casas, 2001; Godoy, 2006; Liberman \& Wolf, 1990), es indispensable tomar en cuenta la institucionalización disciplinaria que, siguiendo el aporte clásico de Burton Clark (1992), se considera aún más importante que la primera para el análisis de la estructuración del campo académico. En el plano de la institucionalización social, y con mayor razón en el plano cognoscitivo, la constitución de una disciplina o especialidad científica atraviesa los establecimientos vinculándolos (y desvinculándolos) entre sí mediante la acción de los sujetos adscritos a ellos (Fuentes, 2006).

El estudio realizado en la primera mitad de la década de los noventa (Fuentes, 1998) sobre la emergencia del campo académico de la comunicación en México supuso un ejercicio de apropiación crítica de aportes teórico-metodológicos de Bourdieu (1975, 1988), Giddens (1984, 1989), Thompson (1990) y otros autores, y se desarrolló

4 Sigue siendo pertinente aclarar que el término "campo", como muchos otros empleados en las ciencias sociales y en las humanidades, y en la revisión misma de las condiciones de desarrollo de sus especialidades, es peligrosamente polisémico. Entre sus diversas acepciones, se ha utilizado traducido del inglés (field) para denominarlo de manera simple "territorialmente" un área de estudio o una "disciplina". Pero para apoyar procesos analíticos más profundos, resulta preferible el empleo de la acepción que en francés (champ) le ha dado Bourdieu, como espacio sociocultural de posiciones objetivas donde los agentes luchan por la apropiación del capital común. El concepto de "campo académico" (homologable al de "campo científico") sirve para captar analíticamente las relaciones entre los sistemas de representaciones que subyacen a las prácticas de sujetos empíricos. Estas prácticas, a su vez, constituyen el propio campo como "espacio" de tensiones entre sujetos, entre sujetos y estructura, y entre la estructura y el entorno sociocultural en que se constituye tal campo (Fuentes \& Vidales, 2011, pp. 44-46). 


\section{DISERTACIONES}

ENSAYOS

Anuario electrónico de estudios en Comunicación Social

ISSN: $1856-9536$

Doi: dx.doi.org/10.12804/disertaciones.09.02.2016.01

Volumen 9, Número 2 / julio-diciembre 2016

Versión PDF para imprimir desde

mediante la construcción de modelos heurísticos, uno de los cuales definió nueve procesos de estructuración ${ }^{5}$, a reconstruir de manera analítica:

Escala individual:

- Procesos de constitución de los sujetos (trayectorias académicas, opciones vocacionales, orígenes sociales)

- Procesos de formación/conformación del habitus (esquemas de percepción, valoración y acción)

- Procesos de profesionalización (como apropiación de recursos y esquemas de competencia académica y como calificación y ubicación laboral en una institución específica)

Escala institucional:

- Procesos de institucionalización social u organización (como programas institucionales y formación de una "comunidad científica" por medio de asociaciones y publicaciones académicas).

- Procesos de institucionalización cognoscitiva (conformación de una "matriz disciplinaria" articuladora y generadora del sentido de las prácticas científicas legítimas)

- Procesos de especialización de la producción científica (en términos de intercambios intra- e interdisciplinarios).

Escala sociocultural:

- Procesos de autoreproducción del propio campo, mediante la formación e incorporación de investigadores en el mercado laboral académico.

- Procesos de legitimación social del campo ante el Estado y la sociedad civil, manifiestos en la obtención de "autoridad científica" y de "autonomía relativa" y en los usos sociales de sus productos.

- Procesos de asimilación/acomodación del sentido (utópico) del campo y de las prácticas en el cambiante entorno sociocultural de la "realidad" (Fuentes, 1998).

Hipotéticamente, mediante la articulación empírica y analítica de reconstrucciones e interrelaciones concretas de uno o varios de estos procesos, es posible reconocer en diferentes escalas espaciales y temporales los factores determinantes de la estructuración/desestructuración/reestructuración del campo académico, situando en sus diferentes contextos las prácticas constitutivas, que incluso pueden llegar a formularse en términos de comunicación. En este estudio, la dimensión transinstitucional es importante, y lo es más, obviamente, cuando las instituciones y los sujetos están situados en distintos países, es decir, en distintos regímenes nacionales. Pero está todavía por escribirse sobre esta u otra lógica, una historia internacional de los estudios de la comunicación

5 Hay que hacer notar el hecho de que, habiendo sido propuesto y publicado (en español) varios años antes, la lógica de este modelo se parece a la subyacente en el trabajo de Robert T. Craig (2008): "Los recursos retóricos para construir y legitimar disciplinas pueden encontrarse en contextos de historia intelectual, institucional y sociocultural" (pp.8-9). Lo mismo podría señalarse, con mayor claridad aún, en referencia al modelo de Löblich \& Scheu (2011) y sus "funciones heurísticas", basadas en la sociología de la ciencia, para interrelacionar, en diferentes niveles teóricos y también en un meta-nivel de investigación, a los "académicos individuales, las instituciones académicas y los campos no científicos de la sociedad" (Löblich \& Scheu, p. 16). 


\section{DISERTACIONES}

Ensayos

Anuario electrónico de estudios en Comunicación Social

ISSN: $1856-9536$

Doi: dx.doi.org/10.12804/disertaciones.09.02.2016.01

Volumen 9, Número 2 / julio-diciembre 2016

Versión PDF para imprimir desde

http://revistas.urosario.edu.co/index.php/disertaciones

y de los medios, pues "hasta ahora, la mayor parte de las historias han sido nacionales, con una predominante atención sobre América del Norte y Europa Occidental”" (Simonson \& Peters, 2008, p. 764).

No obstante, emerge y se fortalece un punto de vista internacional que "nos ayuda a ver cómo el estudio organizado de la comunicación al mismo tiempo ha reflejado, refractado e impulsado la geopolítica transnacional, los patrones institucionales de educación y profesionalización y maneras de conocer y de actuar" (Simonson \& Park, 2016, p.2) determinantes de la vida colectiva desde el siglo pasado. La búsqueda de marcos sociohistóricos adecuados para fundamentar una investigación no solo inter-nacional, sino crecientemente trans-nacional de los procesos de constitución del campo académico de la comunicación, con fundamentos propiamente teóricos, ha cobrado recientemente un fuerte impulso: "Reconociendo que los campos no están limitados por las fronteras de los estados-nación, los historiadores de las ciencias humanas han desarrollado marcos transnacionales de análisis. La historia transnacional toma forma junto a las historias comparativa, internacional, mundial y global" (Simonson \& Park, 2016, p. 6).

\section{La construcción de la inter- o transnacionalidad del campo}

Para un impulsor tan destacado tanto de la conjunción práctica como del reconocimiento conceptual e historiográfico del establecimiento de redes (networking) internacionales como Kaarle Nordenstreng (2008), es notable "lo poco y lo tarde que las instituciones internacionales han intervenido en la conformación de la investigación de la comunicación” (p. 225), hasta la constitución en 1957 de la AIERI/ IAMCR/ AIECS ${ }^{7}$, promovida por la Organización de las Naciones Unidas para la Educación, la Ciencia y la Cultura (Unesco). Pero,

Geopolíticamente, IAMCR tuvo una base amplia, incluso global, afiliando instituciones e individuos de todos los continentes. No hay duda de que la iniciativa de crear IAMCR estuvo dominada por europeos, particularmente por franceses, pero también intervinieron colegas de países como Brasil, Perú, Uruguay, Egipto, Israel, India, Indonesia, Japón, Australia, Estados Unidos y Canadá. La nueva Europa oriental, detrás de la llamada Cortina de Hierro, estuvo representada por líderes académicos provenientes de Polonia, Checoeslovaquia y

6 Pooley y Park (2013) enumeran al final de una acuciosa revisión de más de 1600 documentos publicados (solamente en inglés) sobre la historia del campo, una serie de sesgos que la internacionalización (o "des-Occidentalización" como ellos dicen) de la investigación histórica deberá superar paulatinamente: hay muchos más estudios con enfoques "campo-céntricos/ intelectuales" que "institucionales/ contextuales"; la abrumadora mayoría se refiere a Norte América y Europa; pocos estudios comparan tradiciones nacionales, y menos aún intentan ser historias internacionales; algo hay sobre "campos-dentro-del campo", pero casi nada sobre sus interrelaciones; hay muy pocas historias sobre instituciones concretas y lo mismo puede decirse sobre las biografías personales. "Queda mucho por hacerse, a pesar de la abundancia de publicaciones. Hasta ahora la impresión que tenemos es la de una pintura puntillista a medio terminar: desde lejos se alcanzan a discernir algunas formas, rodeadas de áreas de lienzo sin pintura" (pp. 85-86).

7 Association Internationale des Études et Recherches sur l'Information et la Communication (AIERI), International Association for Media and Communication Research (IAMCR), Asociación Internacional de Estudios en Comunicación Social (AIECS). Nótese, de paso, la diversa denominación del "objeto" de la asociación en sus tres idiomas oficiales. IAMCR (como se le referirá aquí) en la actualidad agrupa miembros institucionales de 36 países y casi 2000 individuos de más de 90 países de todas las regiones del mundo. 


\section{DISERTACIONES}

Ensayos

Anuario electrónico de estudios en Comunicación Social

ISSN: $1856-9536$

Doi: dx.doi.org/10.12804/disertaciones.09.02.2016.01

Volumen 9, Número 2 / julio-diciembre 2016

Versión PDF para imprimir desde

http://revistas.urosario.edu.co/index.php/disertaciones

la Unión Soviética [...] En consecuencia, IAMCR no fue un proyecto de la Guerra Fría. Por el contrario, fue fun-

dada sobre suelo ecuménico, cruzando tanto la brecha Este-Oeste como la brecha Norte-Sur (Nordenstreng,

2008, p. 229).

Indudablemente, después de más de cincuenta años de operación y de cuarenta y dos conferencias (reuniones primero bienales y más recientemente anuales), celebradas en países de todas las regiones y condiciones de desarrollo ${ }^{8}$, IAMCR es un referente institucional imprescindible para el campo de la investigación de la comunicación y Nordenstreng (2008) afirma que ha cumplido adecuadamente su misión, conforme el campo "se ha expandido no solo en términos de instituciones nacionales sino también de redes internacionales", aunque no puede "ser celebrada como un factor decisivo para la internacionalización del campo", pues "la asociación ha seguido, más que impulsado, el desarrollo" (p. 245).

De cualquier manera,

IAMCR ha cumplido un papel vital en la movilización de la dimensión internacional del campo, especialmente en sus primeras décadas. Es poco probable que cualquier otra agrupación pudiera haber promovido más efectivamente el establecimiento de redes en un campo tan profundamente enraizado en condiciones políticas, económicas y culturales nacionales. Esto es un problema de investigación para la sociología de la ciencia, pero en cualquier caso se puede concluir que una lección abarcante de la historia de IAmCR es una paradoja o una ironía: el hecho de que su relativa importancia parece haber disminuido conforme el campo se ha desarrollado y, más particularmente, se ha internacionalizado (Nordenstreng, 2008).

Como lo proponen Michael Meyen (2016) y otros investigadores de estos procesos, entre los factores a analizar en la historia internacional del campo académico de la comunicación, está la politización que, cerca de la Unesco (Wagman, 2016) y con deliberado acento en líneas "críticas" de trabajo como las políticas de comunicación y la regulación de los medios o la democratización y los derechos humanos, ha caracterizado a IAmCR, más que a otras organizaciones, como la International Communication Association (ICA). Esta politización hizo todavía menos probable, pensando bourdianamente, que "IAMCR se convirtiera en el único o principal locus internacional de la 'lucha competitiva' por la autoridad científica y por la definición legítima del propio campo" (Meyen, 2016, p. 92), sin que eso implique escatimar la importancia de su influencia en la definición de los parámetros transnacionales del campo. Para las asociaciones académicas latinoamericanas (ALAIC y FELAFACS), IAMCR fue y es todavía, sobre todo para la primera, un referente fundamental.

Más en general, Simonson y Park (2016) aseveran que las asociaciones académicas transnacionales "han sido las principales fuerzas para facilitar el flujo de ideas y personas, consolidar los paradigmas hegemónicos y contrahegemónicos y las orientaciones políticas de la investigación de la comunicación y de las redes sociales de académicos". Al mismo tiempo advierten la importancia particular que han tenido las asociaciones regionales, sobre todo en América Latina, África, los países árabes y la región nórdica (pp. 69-70). Por su parte, en un Encuentro Internacional de Asociaciones Académicas de Comunicación celebrado en Bilbao en enero de 2014, Miquel

8 Incluyendo la realización de diez de las 42 conferencias en países iberoamericanos: España (1968 Pamplona; 1988 y 2002 Barcelona), Portugal (2010 Braga), Argentina (1972 Buenos Aires), Venezuela (1980 Caracas), Brasil (1992 Guaruja SP y 2004 Porto Alegre) y México (1997 Oaxaca y 2009 Ciudad de México). 


\section{DISERTACIONES}

de Moragas (2014) resumió en un "decálogo" las funciones atribuibles a estas organizaciones, transnacionales, regionales o nacionales:

1. Facilitar el intercambio científico entre expertos y grupos de investigación;

2. Dar visibilidad y legitimidad al campo de estudios de la comunicación;

3. Facilitar la creación de grupos inter-centros;

4. Creación de nodos y gestión de la globalidad;

5. Neutralizar las influencias de los agentes académicos hegemónicos (fundaciones, editoriales, grupo selecto de universidades);

6. Plataforma para la obtención de méritos académicos;

7. Revitalizar la comunidad investigadora;

8. Facilitar el intercambio generacional;

9. Asesoramiento institucional en política científica y en política de comunicación;

10. Confrontar el campo académico con el compromiso social (p. 8).

El espíritu subyacente en esta propuesta, presentada a nombre de la Asociación Española de Investigación de la Comunicación (AE-IC) por su presidente, enfatiza la cooperación académica para enfrentar la competitividad que "las lógicas de la política científica actual tienden a priorizar" y no entender a la globalización como unificación sino como "interconexión de nodos de influencia". En ese sentido, subraya que "las asociaciones nacionales o regionales como ECREA [European Communication Research and Education Association], ALAIC o Confibercom [Confederação Ibero-Americana das Associações Científicas e Acadêmicas de Comunicação] afrontan el grave riesgo de aislacionismo si no saben resituarse en la red de cooperación, si su dinámica no se ve completada con otros nodos de relación (networking) internacional" (Moragas, 2014, pp. 6 y 11). Al seguir estas pistas y otras confluyentes, resulta cada vez más claro que el enfoque de la atención sobre las tensiones y contraposiciones es mucho más esclarecedor de los procesos de institucionalización internacional que los flujos unidireccionales de influencias o recursos, o la defensa de excepcionalismos históricos nacionales, y que metodológicamente conviene definir escalas tanto espaciales como temporales para contextualizar adecuada y diversamente los procesos de la transnacionalización (Löblich \& Averbeck-Lietz, 2016).

\section{Constitución y reconstitución de ALAIC}

La más importante de las asociaciones académicas de la comunicación en América Latina, en cuanto a las funciones que ha cumplido, es la ALAIC, constituida en 1978 y reconstituida en 1989. José Marques de Melo, siendo su presidente (1989-1992), resumió su historia inicial de la siguiente manera:

La ALAIC fue fundada en Caracas en noviembre de 1978, por iniciativa de un grupo de investigadores de la comunicación (Antonio Pasquali, Luis Ramiro Beltrán, Jesús Martín Barbero, Eleazar Díaz Rangel, entre otros), comprometidos con el avance de esta disciplina académica en América Latina. La principal conquista de ALAIC fue la legitimación de la nueva área del conocimiento dentro de la Unesco y las agencias internacionales de fomento científico. Hasta entonces, las investigaciones de la comunicación se confundían con los estudios realizados bajo los cánones de las ciencias sociales, principalmente la sociología. Gracias a la actuación de ALAIc, la comunicación fue reconocida como un área autónoma de investigación 


\section{DISERTACIONES}

Ensaros

Anuario electrónico de estudios en Comunicación Social

ISSN: $1856-9536$

Doi: dx.doi.org/10.12804/disertaciones.09.02.2016.01

Volumen 9, Número 2 / julio-diciembre 2016

Versión PDF para imprimir desde

http://revistas.urosario.edu.co/index.php/disertaciones

académica, naturalmente sin rechazar la articulación interdisciplinaria, una característica intrínseca de

las humanidades (Marques de Melo, 1991, p. 100).

Un año antes, en 1977, varios periodistas y académicos promovieron la constitución de una asociación de investigadores en Venezuela ${ }^{9}$, inspirada, de alguna manera, en lo que habían encontrado participando en AEIRI (y con vistas a la organización en Caracas de la conferencia correspondiente a 1980), para promover el desarrollo de perspectivas críticas sobre los diversos aspectos de la relación entre Comunicación y Cultura de Masas (título del libro pionero de Antonio Pasquali, 1963) y como secuela complementaria de la fundación en 1974 del Instituto de Investigaciones de la Comunicación (ININCO) de la Universidad Central de Venezuela ${ }^{10}$. En 1976, a su vez, se fundó en México el Instituto Latinoamericano de Estudios Transnacionales (ILET), que durante la siguiente década aportó influyentes insumos, producidos por un grupo selecto de exiliados sudamericanos, para una comprensión crítica de diversos aspectos (políticos, económicos y culturales) de la organización, precisamente transnacional, de ciertas actividades estratégicas, entre las que se encontraban incluidas las de "comunicación social"1.

Resulta claro, entonces, que antes que la "legitimación académica" ante la Unesco o la definición disciplinaria de los estudios sobre la comunicación, la alalc fue fundada y se desarrolló en sus primeros años como un foro de acción contra-hegemónica y al mismo tiempo de cooperación intra-regional, en un contexto sociopolítico de resistencia al autoritarismo, particularmente a los gobiernos militares del Cono Sur, y a lo que entonces fue denominado como imperialismo cultural. Ante la escasez y fragilidad de instituciones universitarias y centros académicos de investigación de la comunicación en América Latina, durante las décadas de los sesenta, setenta y ochenta, la investigación y la "teoría militante" que caracterizó a la región fueron fuertemente internacionales, o incluso transnacionales, pero estuvieron muy alejadas de los cánones más ortodoxos de la institucionalización científico-académica y de la enseñanza universitaria. En palabras de Luis Gonzaga Motta (1989), en América Latina, en el área de la comunicación y la cultura,

9 Asociación Venezolana de Investigadores de la Comunicación (Avic), años después reconstituida como Investigadores Venezolanos de Comunicación (InveCom).

10 Según su director en 2014, Carlos Enrique Guzmán Cárdenas, la confluencia en el Ininco de pensadores como Antonio Pasquali (filósofo), Luis Aníbal Gómez (filósofo y periodista), Oswaldo Capriles Arias (abogado), Alfredo Chacón (antropólogo y sociólogo), Federico Álvarez Olivares (periodista), Elizabeth Safar Ganahl (comunicadora social), Oscar Lucien (sociólogo y cineasta), Gustavo Alfredo Hernández Díaz (licenciado en artes y educador) y otros, "favorecerá un movimiento de perspectiva cultural sobre los medios de comunicación, basado en las Ciencias Sociales” (comunicación personal, 2014). Ese movimiento influiría decisivamente en los años siguientes sobre otras instituciones venezolanas y latinoamericanas como la AVIC y la ALAIC.

11 Marques de Melo resalta "la amplitud temática y la calidad académica de los trabajos realizados por el ILET", lo que lo convirtió en "la principal institución latinoamericana difusora de propuestas alternativas para la democratización de los medios de comunicación en el hemisferio". Sus investigadores, especialmente los chilenos Juan Somavía, Fernando Reyes Matta y Diego Portales, el argentino Héctor Schmucler y el peruano Rafael Roncagliolo, tuvieron "una presencia destacada en los foros regionales e internacionales, promocionando sobre todo las tesis del Nuevo Orden Mundial de la Información y la Comunicación" (Marques de Melo, 1989, p.153) y, habría que agregar, fueron determinantes, junto a los venezolanos, en la fundación y primer desarrollo de la ALAIC. 


\section{DISERTACIONES}

No ha tenido lugar la institucionalización weberiana de la ciencia (consagración de comportamientos de la 'comunidad científica' a través de la asimilación de papeles sociales propios de la ciencia, tales como el desinterés político, la racionalidad y la neutralidad emotiva), al menos con los moldes norteamericanos. En realidad, la actividad científica en materia de comunicación durante las últimas tres décadas (tal vez con la excepción de Brasil), no se ha institucionalizado ni siquiera en cuanto a la instalación de un lugar propio y aceptado como lugar de investigación. En realidad, la producción científica más significativa de esta área se realizó, y todavía se realiza, fuera de los mecanismos del estado (universidades, tecnoburocracia, etc.) (Gonzaga Motta, 1989, pp. 150-151).

Bajo las presidencias entreveradas de los venezolanos Luis Aníbal Gómez y Oswaldo Capriles (sustituido al final de su gestión por Alejandro Alfonzo) y los colombianos Jesús Martín Barbero y Patricia Anzola, la alaic impulsó la creación de asociaciones nacionales de investigadores de la comunicación y promovió el levantamiento de la bibliografía contemporánea sobre la investigación de la comunicación en varios países de la región ${ }^{12}$. Estas, entre otras iniciativas de institucionalización académica y cooperación internacional, convirtieron a la ALAic en el núcleo central de una convergencia intelectual y política que Robert White describía en 1989 como una tendencia general a relacionar la investigación sobre la comunicación "con problemas básicos que se refieren a la clase de sociedad y cultura que está emergiendo en América Latina y a cuál es el papel que los medios de comunicación deberían jugar en ese proceso" (White, 1989, p. 44). El cambio político y social, la erradicación de las estructuras impuestas por regímenes autoritarios y la dependencia de poderes externos, la expresión de las culturas populares y la democratización de los medios de comunicación de masas fueron asumidos como condiciones generalizadas y referentes transnacionales en la región, que fue a su vez reconocida como una unidad geopolítica y cultural donde se gestaba un "pensamiento crítico propio" y hasta una "Escuela Latinoamericana" de estudios de la comunicación (León, 2006; Gobbi, 2008). No obstante, desde mediados de la década de los ochenta, las actividades de aLAIC disminuyeron en intensidad y alcance, al grado que debió iniciarse en 1988 un proceso de reconstitución sobre nuevas bases e impulsos:

La crisis vivida por ALAIc no constituyó un hecho aislado, contándose en la deuda social responsable de la configuración de lo que se dio en llamar la "década perdida". Felizmente, la acción de destacadas personalidades de la comunidad (entre los cuales están Rafael Roncagliolo, Luis Peirano, Jesús Martín Barbero, Anamaria Fadul, Fátima Fernández y Joaquín Sánchez) impidió la desagregación de ALAlc. Reunidos informalmente en Barcelona, en julio de 1988 durante el xVI Congreso de la AIERI [IAMCR], cerca de veinte latinoamericanos asumieron la causa de la reconstitución de ALAIC [...] En diciembre de 1988 se reunieron en la ciudad paulista de Embu-Guaçu representantes de las principales organizaciones brasileñas y mexicanas

12 Ese proyecto de documentación científica actualizó y complementó en los años ochenta lo que CIESPAL había desarrollado desde la década de los sesenta y que permitió recuentos y diagnósticos críticos "fundacionales" sobre la investigación de la comunicación en América Latina, como el célebre estudio de Luis Ramiro Beltrán (1974) presentado en una conferencia de IAMCR. Lamentablemente, y a pesar de la extensión que han alcanzado los recursos tecnológicos informacionales, no se ha vuelto a coordinar en el campo un esfuerzo continental equivalente (Fuentes, 2014a). 


\section{DISERTACIONES}

Ensayos

Anuario electrónico de estudios en Comunicación Social

ISSN: $1856-9536$

Doi: dx.doi.org/10.12804/disertaciones.09.02.2016.01

Volumen 9, Número 2 / julio-diciembre 2016

Versión PDF para imprimir desde

http://revistas.urosario.edu.co/index.php/disertaciones

del área de la comunicación (INTERCOM, ABECOM, UCBC, AMIC, CONEICC ${ }^{13}$ ) y de la OCIC/AL ${ }^{14}$, firmándose entonces una convocatoria a la Asamblea de Reconstitución de ALAIC [...] [que] se realizó en la ciudad de Florianópolis (Santa Catarina, Brasil), el 8 de septiembre de 1989 [...] contando con representantes de 12 países (Argentina, Bolivia, Brasil, Chile, Colombia, Cuba, México, Nicaragua, Perú, Puerto Rico, Uruguay y Venezuela) (Marques de Melo, 1991, p. 101).

La ALAIC reconstituida, a diferencia de su predecesora, ha realizado funciones más comúnmente reconocidas como propias de una organización académica internacional y su institucionalización ha soportado la prueba de la renovación periódica de sus directivos ${ }^{15}$, del fortalecimiento y ampliación de proyectos conjuntos con otras asociaciones regionales y globales del campo (IAMCR, ICA, ECREA y otras), de la organización bienal de congresos y seminarios internacionales ${ }^{16}$, y del sostenimiento de dos revistas científicas (Revista Latinoamericana de Ciencias de la Comunicación, en portugués y español desde 2004 y Journal of Latin American Communication Research, en inglés, desde 2011). A pesar de la creciente desproporción entre Brasil y el resto de los países latinoamericanos en la cantidad de participantes calificados y los grados de institucionalización del campo de la comunicación (Vassallo de Lopes \& Romancini, 2016), ALAIC ha logrado consolidarse como un espacio latinoamericano abierto tanto a las muy diversas realidades nacionales y locales de la región como al contacto y colaboración con organismos académicos de otras regiones del planeta.

\section{FELAFACS, reproducción y renovación educativas del campo}

Aunque su ámbito de acción no es estrictamente la investigación, feLAFACS ha sido también, desde su constitución en octubre de 1981, un organismo de apoyo y difusión académicos a la investigación latinoamericana y una instancia determinante para la institucionalización del campo académico, al agrupar a los programas de formación universitaria, especialmente de pregrado, y sus asociaciones nacionales y articularlos mediante proyectos conjuntos, de escala regional. Las actividades de felafacs han sido muy variadas y han abarcado a los veinte países de la regió $\mathrm{n}^{17}$, gracias en parte a que durante muchos años contó con el respaldo de la Fundación Konrad Adenauer, de la Unesco y de otras instituciones, y con la destacada actuación de Joaquín Sánchez como su primer presidente

13 Sociedade Brasileira de Estudos Interdisciplinares da Comunicação; Associação Brasileira de Faculdades de Comunicação Social; União Cristã Brasileira de Comunicação Social; Asociación Mexicana de Investigadores de la Comunicación; Consejo Nacional para la Enseñanza y la Investigación de las Ciencias de la Comunicación.

14 Organización Católica Internacional de Cine para América Latina.

15 Desde 1989 alaic ha sido presidida por tres brasileños: José Marques de Melo, Margarida Kunsch y César Bolaño; dos mexicanos: Enrique Sánchez Ruiz y Delia Crovi; el peruano Luis Peirano y el boliviano Erick Torrico, cada uno acompañado con consejos directivos multinacionales.

16 Entre 1992 y 2016, ALAIC ha organizado trece congresos latinoamericanos: 3 en Brasil, 3 en México y en Venezuela, Chile, Bolivia, Argentina, Colombia, Uruguay y Perú, así como nueve seminarios internacionales, de periodicidad irregular, a partir de 1999: 3 en Bolivia, 2 en Brasil, 2 en Argentina, en Venezuela y en Puerto Rico.

17 Son actualmente miembros plenos de felafacs ocho asociaciones nacionales y 34 universidades ubicadas en países donde no hay asociación nacional, para un total cercano a las 240 instituciones, además de diez universidades españolas, dos estadounidenses y una canadiense como miembros asociados, y 


\section{DISERTACIONES}

y de Walter Neyra como su secretario ejecutivo. En un documento inédito, "Informe Evaluativo" sobre la fELAfacS Wolfgang Donsbach (1997) describió tres funciones básicas, "manifiestas y latentes", cumplidas por la Federación:

En primer lugar, la organización es un foro y una red comunicacional para las facultades latinoamericanas de comunicación social. En esa función, la organización crea algo así como una identidad común de las facultades latinoamericanas de comunicación social. En segundo lugar, felafAcs es una institución educativa, dedicada sobre todo a la formación de los formadores. En este sentido, la organización crea calidad. Esta calidad se refiere, por una parte, al trabajo docente y de investigación y, por el otro, al nivel de graduados de las carreras representadas en fELAFACS. En tercer lugar, fELAFACS cumple la función de una institución que promueve a la investigación. En esta calidad, fELAFACS puede contribuir a la obtención de mayores conocimientos sobre fenómenos específicos latinoamericanos y problemas en el ámbito de la comunicación pública (Donsbach, 1997, p. 76).

Después de la realización de dos Encuentros Latinoamericanos de Facultades de Comunicación Social organizados por la Universidad de Lima en 1979 y 1980, en los que se gestó la fELAFACS, esta ha realizado trece encuentros más, cada dos o tres años, dependiendo de las conveniencias de las sedes, debido a la nutrida participación de estudiantes y profesores, lo que implica una logística más compleja que un congreso o un seminario de investigadores ${ }^{18}$.

Conviene aquí recuperar algunos antecedentes relacionados con la enseñanza de la comunicación, antes de 1950 se habían fundado doce escuelas de periodismo en América Latina: dos en Argentina, Brasil, Ecuador y México, y en Perú, Venezuela, Colombia y Cuba, programas todos que surgieron esencialmente como respuesta a una necesidad de "modernización y desarrollo" de las prácticas periodísticas por medio de la profesionalización de los informadores. Durante los años cincuenta y sesenta este modelo de formación universitaria, a pesar de las diferentes especificidades de cada institución, adquirió cierta solidez: pretendía capacitar técnica y culturalmente a los reporteros y operadores de la prensa escrita y lo lograba en buena medida, relegando a un segundo término el trabajo teórico y el cuestionamiento del contexto social en el que el periodismo habría de ejercerse. Pero el afán modernizador de la "Alianza para el Progreso" y proyectos similares, adoptados por los países latinoamericanos, desbordaba paulatinamente a la prensa, que además acusaba vicios y obstáculos tradicionales sobre los cuales afincó su industrialización, y ponía en un lugar muy relevante a la radio y la televisión (Fuentes, 1989).

Desde finales de la década de los cincuenta, las necesidades de impulso a la información social, definidas desde la ideología imperante, se desplazaron, ampliándose, hacia los entonces “nuevos” medios electrónicos. Para 1960 funcionaban ya 44 escuelas de periodismo en América Latina, situadas en Argentina (8), Brasil (8), Cuba (6), México (6), Colombia (3), Perú (3), Ecuador (2), Venezuela (2), Chile, República Dominicana, El Salvador, Guatemala, Nicaragua y Panamá (Nixon, 1982). En ese año, se fundó la licenciatura en Ciencias de la Comunicación

16 organizaciones y 14 personas como miembros honorarios. Han sido sus presidentes tres colombianos, un mexicano y una peruana.

18 Por esa razón, se ha mantenido la denominación de "Encuentros" (y no "Congresos" o "Conferencias"), en los que el programa propiamente académico es complementado con otras actividades, algunas de ellas masivas, dirigidas a estudiantes. Se han realizado cuatro de estos Encuentros en Colombia (1986, 1994, 2006 y 2015), dos en Perú (1997 y 2012, además de los previos, 1979 y 1980), dos en México (1982 y 1992), dos en Brasil (1984 y 2000), y en Panamá (1989), Puerto Rico (2003) y Cuba (2009). 


\section{DISERTACIONES}

ENSAYOS

Anuario electrónico de estudios en Comunicación Social

ISSN: 1856-9536

Doi: dx.doi.org/10.12804/disertaciones.09.02.2016.01

Volumen 9, Número 2 / julio-diciembre 2016

Versión PDF para imprimir desde

http://revistas.urosario.edu.co/index.php/disertaciones

(Ciencias y Técnicas de la Información), en la Universidad Iberoamericana en la ciudad de México ${ }^{19}$ y comenzó el crecimiento exponencial del número de instituciones y de estudiantes en todos los países latinoamericanos. Nixon (1982) enlistó 81 escuelas en 1970 y 163 en 1980, dos tercios de las cuales se ubicaban en Brasil y en México, los dos países con mayor población y con industrias mediáticas más consolidadas. En ese contexto se constituyó la FELAFACS.

Aunque por supuesto sería incongruente tratar de entender a las universidades latinoamericanas como actualizaciones de un modelo homogéneo, es un hecho que en mayor parte están orientadas por la herencia napoleónica de formación "profesionalizante", lo cual explicaría en buena medida la generalización de ciertas características de las carreras de comunicación: la dependencia en su orientación de la estructura comercial hegemónica en los medios "masivos" y la desvinculación de la docencia y la investigación. Los posgrados, para la formación en investigación, se han desarrollado tardía y desbalanceadamente en América Latina (aunque en 2011 sumaban 249 maestrías y 38 doctorados en comunicación en 19 países), y constituyen un ámbito que felAFAcs no ha atendido y que solo en Brasil ha generado la agrupación de los programas de este nivel en una asociación específica, llamada compós ${ }^{20}$ (Vassallo de Lopes, 2012; Vassallo de Lopes \& Romancini, 2016). Por diversas razones, algunas de las cuales provienen precisamente del avanzado grado de institucionalización alcanzado, ninguna asociación de programas representa actualmente a Brasil en fELAFACS.

En suma, puede afirmarse que en relación con sus objetivos originales, fELAFAcs ha sido rebasada por el crecimiento del número de escuelas y de estudiantes de comunicación, aunque también por el predominio generalizado de proyectos de formación profesional muy distintos a los que motivaron su constitución y por la transformación misma de los sistemas de comunicación social en los contextos locales, nacionales y transnacionales. No obstante, los Encuentros latinoamericanos, los innumerables talleres y seminarios organizados por la Federación y el sostenimiento de la publicación de la revista Diálogos de la Comunicación ${ }^{21}$ han permitido articular la problemática y los recursos de la enseñanza y, en menor medida, la investigación de la comunicación en torno a situaciones sociales concretas, muchas de ellas muy cambiantes y complejas.

\section{5. ¿Internacionalización desintegrada y unión fragmentada?}

Según Karl Erik Rosengren, el eje de las discusiones centrales del campo académico de la comunicación se desplazó, en la década de los ochenta, de la dimensión cambio radical/regulación social (es decir, un eje orientado por ideologías políticas), a la dimensión subjetivismo/objetivismo (a su vez definido más bien por ideologías científicas). Pero, al mismo tiempo y, quizá por ello, el campo "se caracteriza hoy más por la fragmentación que por la fermentación” (Rosengren, 1994). A partir de entonces, la preocupación por la fragmentación, entendida como

19 Este nuevo modelo, humanístico, de formación universitaria se extendería a otras universidades a partir de la década de los setenta, coincidiendo con un movimiento continental impulsado por CIESPAL para convertir a las escuelas de periodismo en facultades de comunicación (social) (Fuentes, 1989, 2016).

20 Associação Nacional dos Programas de Pós-Graduação em Comunicação, fundada en 1991.

21 En versión impresa: 57 números publicados de 1987 a 2007 (después de 16 números de Boletín), y en formato digital desde entonces, hasta el número 92 en 2016 [http://dialogosfelafacs.net/]. 


\section{DISERTACIONES}

ENSAYOS

Anuario electrónico de estudios en Comunicación Social

ISSN: 1856-9536

Doi: dx.doi.org/10.12804/disertaciones.09.02.2016.01

Volumen 9, Número 2 / julio-diciembre 2016

Versión PDF para imprimir desde

http://revistas.urosario.edu.co/index.php/disertaciones

obstáculo para la consolidación disciplinaria, se ha convertido en una clave constante y generalizada de discusión, sobre la base de la observación de marcos bibliográficos y referenciales cada vez más especializados y la conformación de sub-campos con menor contacto y debate entre ellos (Bryant \& Miron, 2004). En América Latina, además, esta dispersión temática y teórico-metodológica, perceptible en los congresos, encuentros y conferencias y en las bibliografías de tesis de posgrado (Fuentes, 2008), coexiste con una creciente concentración de la producción y la distribución de contribuciones científicas, sin que esto signifique una paradoja o una contradicción, como se ha argumentado en otro lugar:

A manera de ilustración (y quizá como sugerencia de análisis que deberían realizarse), pueden tomarse, por ejemplo, los datos expuestos por Ángela María Godoy (2006), quien sistematizó y analizó los orígenes de 447 artículos publicados en 51 ediciones de la revista Diálogos de la Comunicación, entre 1987 y 2003. La distribución por adscripción de los autores indica una concentración del $64 \%$ en los cinco países con mayores frecuencias (México, 17\%; Argentina, 12,8\%; Colombia, 12,3\%; Brasil, 11,2\% y Perú, 10,7\%), mientras que otros 10 países latinoamericanos dan cuenta del $18,8 \%$ de los artículos y otros 12 países de otras regiones, del $17,2 \%$ restante. Comparativamente, en las 16 ediciones de la Revista Latinoamericana de Ciencias de la Comunicación publicadas entre 2004 y 2012, los cinco países con mayores frecuencias concentran el 81,9\% de los 176 artículos (Brasil, 47,2\%; México, 14,8\%; Argentina, 10,2\%; España, 5,7\% y Chile, $4 \%$ ). Restan un 9,6\% para otros ocho países latinoamericanos y un $8,5 \%$ para cinco países de otras regiones. Y si se sumaran los artículos, a pesar de las diferencias entre las dos revistas, el 21,3\% correspondería a autores brasileños, el $16,4 \%$ a mexicanos y el $12 \%$ a argentinos, es decir, el $49,7 \%$ del total para solo tres países (Fuentes, 2015, pp. 68-69).

A propósito de la fragmentación, el presidente del Social Science Research Council, Craig Calhoun (2011), afirmó en una Conferencia de la ICA que "en este heterogéneo campo lo que se necesita no es presión hacia la conformidad sino la producción de más y mejores conexiones entre diferentes líneas de trabajo”. La concepción de campo académico adquiere así la mayor pertinencia, pues "la teoría tiene un papel especial que desempeñar en esto, pero hacer las grandes preguntas que conecten diferentes líneas de trabajo es algo que rebasa por mucho el dominio de la teoría" (p. 1495). Y aunque la dimensión teórica no es precisamente la más desarrollada en las instituciones latinoamericanas, hay sin duda alguna una gran experiencia en la formulación y enfrentamiento de los desafíos de la múltiple y desigual realidad social.

Una apreciación similar a la de Calhoun, aunque mucho más detallada y fundamentada es sostenida por Silvio Waisbord (2014), para quien lo que se necesita es adoptar una postura analítica parecida a la que ya generó nuevos avances teóricos en el pasado: "una visión cosmopolita que ubica las cuestiones teóricas en el centro, acercamientos teóricos producidos en diferentes sitios cauta y críticamente asumidos, y comprometida con los debates de la comunidad académica global". Para él, en América Latina "el campo de los estudios de comunicación/medios permanece unido aunque fragmentado" (p. 2), pues a diferencia de lo que sucedió en Estados Unidos - Europa Occidental, emergió de una trayectoria intelectual común y su canon como estudios de la comunicación es más homogéneo intelectualmente, al incorporar "textos clásicos de la tradición del imperialismo cultural y mediático, la semiótica estructural y el análisis del discurso, la filosofía continental, los estudios culturales y la economía política. Estas teorías y acercamientos han sido la lingua franca del campo" (Waisbord, 2014, pp. 6-7), lecturas obligadas en las bibliografías de los programas de formación en América Latina. En consecuencia: 


\section{DISERTACIONES}

ENSAYOS

Anuario electrónico de estudios en Comunicación Social

ISSN: $1856-9536$

Doi: dx.doi.org/10.12804/disertaciones.09.02.2016.01

Volumen 9, Número 2 / julio-diciembre 2016

Versión PDF para imprimir desde

http://revistas.urosario.edu.co/index.php/disertaciones

El campo ha estado abierto a las tendencias intelectuales y de desarrollos globales y regionales. Fue un campo de estudio globalizado en tanto que conectado a los debates intelectuales de todas partes, antes de que la globalización se convirtiera en un término común de la era post-Guerra Fría. Buscó des-Occidentalizar los estudios de comunicación antes de que eso se convirtiera en una preocupación de académicos occidentales. Fue escéptico ante los reclamos universalistas antes de que se pusieran de moda el deconstruccionismo y el postmodernismo. Fue descaradamente cosmopolita, repensando constantemente la viabilidad en la región de las ideas producidas en Europa y Estados Unidos (Waisbord, 2014, pp.7-8).

Además, en palabras de Miquel de Moragas:

No es raro escuchar conferencias, leer ponencias de autores latinoamericanos que se refieren con gran naturalidad a múltiples ejemplos de sus respectivos y numerosos países. La investigación de la comunicación en América Latina no es homogénea, pero se basa en algo muy particular: compartir la diversidad y de-construir los aparatos teóricos sobre comunicación basados en la experiencia ajena de las grandes metrópolis del mundo occidental desarrollado (Moragas, 2011, p. 302).

Si estas apreciaciones, ciertamente optimistas, se ajustan a la descripción y a la prescripción de los patrones de la internacionalización en el campo académico de la comunicación en América Latina, la contribución mediadora de ALAIC y FELAFACS, en cuanto al establecimiento y desarrollo de redes de comunicación académico-social podrá seguirse reconociendo como una "amigocracia" productiva, participativa y creativa, múltiplemente interconectada y comprometida, como Robert White lo imaginó hace más de un cuarto de siglo.

\section{Referencias}

1. Beltrán, L. (1974). Communication Research in Latin America: the blindfolded inquiry? Paper submitted to the International Scientific Conference on Mass Communication and Social Consciousness in a Changing World. Leipzig: International Association for Mass Communication Research.

2. Bourdieu, P. (1975). La specificité du champ scientifique et les conditions sociales du progres de la raison. Sociologie et Societés VII(I), 91-118.

3. Bourdieu, P. (1988). Homo Academicus. Stanford: Stanford University Press.

4. Bryant, J., \& Miron, D. (2004). Theory and Research in Mass Communication, Journal of Communication 54(4), 662-704.

5. Calhoun, C. (2011). Communication as Social Science (and More), International Journal of Communication 5, 1479-1496.

6. Casas, R. (coord.) (2001). La formación de redes de conocimiento. Una perspectiva regional desde México. Barcelona: Anthropos/ IIS Universidad Nacional Autónoma de México.

7. Clark, B. (1992). El sistema de educación superior. Una visión comparativa de la organización académica. México: Nueva Imagen/ Universidad Futura/ uam Azcapotzalco.

8. Craig, R. (2008). Communication in the Conversation of Disciplines, Russian Journal of Communication 1(1), 7-23.

9. Donsbach, W. (1997). Proyecto Federación Latinoamericana de Facultades de Comunicación Social (FELAFACS), Informe Evaluativo, documento inédito. 


\section{DISERTACIONES}

10. Fuentes, R. (1989). El estudio de la comunicación en las universidades latinoamericanas. Telos 19, 156-159.

11. Fuentes, R. (1992). Un campo cargado de futuro. El estudio de la comunicación en América Latina. México: FELAFACS.

12. Fuentes, R. (1998). La emergencia de un campo académico: continuidad utópica y estructuración científica de la investigación de la comunicación en México. Guadalajara: ITEso/ Universidad de Guadalajara.

13. Fuentes, R. (Coord.) (2006). Instituciones y redes académicas para el estudio de la comunicación en América Latina. Guadalajara: ITESo.

14. Fuentes, R. (2008). Bibliografías, biblionomías, bibliometrías: los libros fundamentales en el estudio de la comunicación, Comunicación y Sociedad (nueva época) 10, 15-53.

15. Fuentes, R. (2014a). Recursos para la desfragmentación de la investigación iberoamericana en comunicación, Revista Latinoamericana de Ciencias de la Comunicación 20, 16-25.

16. Fuentes, R. (2014b). La investigación de la comunicación en América Latina: una internacionalización desintegrada, Oficios Terrestres 31, 11-22.

17. Fuentes, R. (2015). Teoría y metodología de la investigación en comunicación en América Latina: ALAIc y el desafío de la fragmentación. En Bolaño, Crovi \& Cimadevilla, (Coords.) La contribución de América Latina al campo de la Comunicación. Historia, enfoques teóricos, epistemológicos y tendencias de la investigación (pp. 55-81). Buenos Aires: Prometeo,.

18. Fuentes-Navarro, Raúl (2016). Institutionalization and Internationalization of the Field of Communication Studies in Mexico and Latin America En Simonson \& Park (Eds.), The International History of Communication Study (pp. 325-345). New York and London: Routledge.

19. Fuentes, R., \& Vidales, C. (2011). Fundaciones y fundamentos del estudio de la comunicación. Monterrey: CECYTE-CAEIP.

20. Giddens, A. (1984). The Constitution of Society. Outline of the Theory of Structuration. Berkeley and Los Angeles: University of California Press.

21. Giddens, A. (1989). The orthodox consensus and the emerging synthesis. En Dervin et al. (Eds.) Rethinking Communication Vol. 1: Paradigm Issues (pp.53-65). Newbury Park CA: Sage.

22. Gobbi, M. (2008). A batalha pela hegemonia comunicacional na América Latina. São Bernardo do Campo, SP: Cátedra unEsco Universidade Metodista de São Paulo.

23. Godoy, Á. (2006). Las redes socioacadémicas. Un análisis de la aportación mexicana. En R Fuentes-Navarro et al. (Coord.), Diá-logos de la Comunicación, Instituciones y redes académicas para el estudio de la comunicación en América Latina (pp. 89-130). Guadalajara: ITEso.

24. Gómez-Palacio, C. (1989). The Origins and Growth of Mass Communication Research in Latin America. PhD unpublished dissertation, Department of Communication, Stanford University.

25. Jensen, J. (1994). The consequences of vocabularies. En Levy and Gurevitch (Eds.), Defining Media Studies (pp. 75-82) Oxford and New York: Oxford University Press.

26. León, G. (2006). Sobre la institucionalización del campo académico de la comunicación en América Latina. Una aproximación a las características estructurales de la investigación latinoamericana en comunicación. Tesis doctoral. Universidad Autónoma de Barcelona. 


\section{DISERTACIONES}

ENSAYOS

Anuario electrónico de estudios en Comunicación Social

ISSN: $1856-9536$

Doi: dx.doi.org/10.12804/disertaciones.09.02.2016.01

Volumen 9, Número 2 / julio-diciembre 2016

Versión PDF para imprimir desde

27. Liberman, S., \& Wolf, K. (1990). Las redes de comunicación científica. Cuernavaca: cRIm Universidad Nacional Autónoma de México.

28. Löblich, M., \& Scheu, A. (2011). Writing the History of Communication Studies: A Sociology of Science Approach, Communication Theory 21(1), 1-22.

29. Löblich, M., \& Averbeck-Lietz, S. (2016). The transnational flow of ideas and histire croisée with attention to the cases of France and Germany. En Simonson \& Park (Eds.), The International History of Communication Study (pp. 25-46). New York and London: Routledge.

30. Marques de Melo, J. (1988). Communication Theory and Research in Latin America: a preliminary balance of the past twenty-five years. Media, Culture \& Society, 10, 405-418.

31. Marques de Melo, J. (1989). Los centros de investigación de la comunicación en América Latina, Telos, 19, 151-156.

32. Marques de Melo, J. (1991). Comunicaçao e Modernidade. Loyola: São Paulo.

33. Marques de Melo, J. (2015). El campo académico de la comunicación en América Latina: perspectiva histórica. En Bolaño, Crovi \& Cimadevilla (Coords.), La contribución de América Latina al campo de la comunicación. Historia, enfoques teóricos, epistemológicos y tendencias de la investigación (pp. 21-38). Buenos Aires: Prometeo.

34. Martín-Barbero, J. (1989). Panorama bibliográfico de la investigación latinoamericana en comunicación 1984-1989, Telos 19, 140-147.

35. Meyen, M. (2016). The IAMCR Story. Communication and Media Research in a Global Perspective. En Simonson \& Park (Eds.), The International History of Communication Study (pp. 90-106). New York and London: Routledge.

36. Moragas, M. (2011). Interpretar la comunicación. Estudios sobre medios en América y Europa. Barcelona: Gedisa.

37. Moragas, M. (2014). Las asociaciones de investigación de la comunicación. Funciones y retos. Ponencia presentada en el Encuentro Internacional de Asociaciones Académicas de Comunicación. Razón de ser y retos en la globalización, Bilbao.

38. Motta, L. (1989). Las revistas de comunicación en América Latina: creación de la teoría militante, Telos 19, 147-151.

39. Nixon, R. (1982). Historia de las Escuelas de Periodismo, Chasqui (segunda época) 2, 13-19.

40. Nordenstreng, K. (2008). Institutional Networking: the Story of the International Association for Media and Communication Research (IAmCR). En Park \& Pooley, The History of Media and Communication Research: Contested Memories (pp. 225-248). New York: Peter Lang.

41. Pasquali, A. (1963). Comunicación y Cultura de Masas. Caracas: Monte Ávila.

42. Pooley, J., \& Park, D. (2013). Communication Research. En Simonson, Peck, Craig \& Jackson Jr. (Eds.) The Handbook of Communication History (pp. 76-90). New York and London: Routledge.

43. Schlesinger, P. (1989). Aportaciones de la investigación latinoamericana, Telos 19, pp.55-60.

44. Simonson, P., \& Peters, J. (2008). Communication and Media Studies, History to 1968. En Wolfgang Donsbach (Ed.), The International Encyclopedia of Communication Vol. II (pp.764-771). New York: Blackwell. 


\section{DISERTACIONES}

ENSAYOS

45. Simonson, P., \& Park, D. (Eds.) (2016). The International History of Communication Study, New York and London: Routledge.

46. Thompson, J. (1990). Ideology and Modern Culture. Critical social theory in the era of Mass Communication. California: Stanford University Press.

47. Vassallo de Lopes, M. (Coord). (2012). Posgrados en Comunicación en Iberoamérica. Políticas Nacionales e Internacionales. São Paulo: ECA-USP/ PPG COM USP/ CONFIBERCOM.

48. Vassallo de Lopes, M., \& Romancini, R. (2016). History of Communication Study in Brazil. The institutionalization of an interdisciplinary field. En Simonson \& Park, The International History of Communication Study (pp. 346-366). New York and London: Routledge.

49. Wagman, I. (2016). Locating unEsco in the Historical Study of Communication En Simonson \& Park, The International History of Communication Study (pp. 71-89). New York and London: Routledge.

50. Waisbord, S. (2014). United and fragmented: Communication and media studies in Latin America, Journal of Latin American Communication Research 4(1), 1-23.

51. White, R. (1989). La teoría de la comunicación en América Latina, Telos 19, 43-54. 